Research Article

\title{
Correlation between the Intensity of Helicobacter pylori Colonization and Severity of Gastritis
}

\author{
Hamid Reza Ghasemi Basir, ${ }^{1}$ Mehdi Ghobakhlou, ${ }^{2}$ Parvin Akbari, ${ }^{3}$ Arash Dehghan, ${ }^{1}$ \\ and Mohamad Ali Seif Rabiei ${ }^{4}$ \\ ${ }^{1}$ Department of Pathology, School of Medicine, Hamadan University of Medical Sciences, Hamadan, Iran \\ ${ }^{2}$ Clinical Research Development Unit of Shahid Beheshti Hospital, Hamadan University of Medical Sciences, Hamadan, Iran \\ ${ }^{3}$ School of Medicine, Hamadan University of Medical Sciences, Hamadan, Iran \\ ${ }^{4}$ Department of Community Medicine, School of Medicine, Hamadan University of Medical Sciences, Hamadan, Iran
}

Correspondence should be addressed to Mehdi Ghobakhlou; dr.mghobakhlou@yahoo.com

Received 26 August 2017; Accepted 19 October 2017; Published 28 November 2017

Academic Editor: Tatsuya Toyokawa

Copyright (c) 2017 Hamid Reza Ghasemi Basir et al. This is an open access article distributed under the Creative Commons Attribution License, which permits unrestricted use, distribution, and reproduction in any medium, provided the original work is properly cited.

Background. The most common cause of chronic gastritis is infection with Helicobacter pylori. Identifying the relationship between intensities of colonization and activity of gastritis helps the clinician in more effective treatment and posttreatment follow-ups. Methods. In this cross-sectional study, endoscopic gastric biopsy samples of 544 patients who complained symptoms of dyspepsia for more than three months referring to the laboratory were studied. To determine the colonization rate of $H$. pylori and other pathological findings, Giemsa and H\&E stains were, respectively, used. Results. Among 544 subjects, 47 (8.64\%) patients had no gastritis, 203 (37.32\%) had mild gastritis, 278 (10.51\%) suffered moderate gastritis, and $16(2.94 \%)$ had severe gastritis. In this study, patients with mild $H$. pylori colonization rates had the highest level of mild activity (33.52\%); in contrast, those with severe $H$. pylori colonization had the highest level of severe activity (43.75\%). 93.96\% of people with severe $H$. pylori colonization suffered from moderate and severe chronic gastritis. There is a significant statistical relationship between the intensity of $H$. pylori colonization and histopathological findings including intestinal metaplasia, atrophy, and lymphoid follicle formation. Conclusions. According to the present study, with increasing intensity of $H$. pylori colonization, chronicity and activity of gastritis and its complications increase.

\section{Introduction}

Today, the most common cause of chronic gastritis is infection with Helicobacter pylori (H. pylori). In addition to active chronic gastritis, this organism has a strong relationship with gastric adenocarcinoma and MALToma [1]. Causes of gastritis are widespread and heterogeneous. Gastritis can be classified according to the duration of the disease (acute or chronic), histological aspects, anatomical distributions, and possible pathogenic mechanisms. Histologically, chronic gastritis is characterized by infiltration of inflammatory cells with the preference of lymphocytes and plasma cells, along with a number of neutraophils, in the lamina propria. Chronic gastritis has three stages. In the first stage, inflammatory changes are limited to the laminar propria of the mucosal surface together with edema and inflammatory infiltration which separate the normal-appearing gastric glands. In the second stage, inflammatory infiltration extends to the deeper areas of the mucosa and is associated with deformation and destruction of the glands. In the third stage, the gland structures are demonstrating atrophic changes and inflammatory infiltrations decrease. Atrophic gastritis along with metaplasia is observed in chronic gastritis due to Helicobacter pylori. This may ultimately lead to gastric adenocarcinoma [2].

Helicobacter pylori is a microaerophilic gram-negative bacillus that is commonly found in the deep parts of the mucous gel covering the gastric mucosa or between the mucous layer and the gastric epithelium. The prevalence of H. pylori is higher in older ages. Two prone factors to 
increase the colonization of this organism include poor socioeconomic status and low education. Nature of the inflammatory response created by $H$. pylori is defined based on bacterial virulence factors, intensity of bacterial colonization, and the host response [3].

Chronic infection with $H$. pylori stimulates the host's immune response, causes active chronic inflammation and mucosal injury resulting in multifocal atrophic gastritis and intestinal metaplasia, glandular dysplasia, and adenocarcinoma [4]. After treatment, the shape of the bacteria may also become round or Vibrio and H. pylori colonization decreases or reaches zero. After eradication of $H$. pylori, histology of the lesion is improved [5].

Considering the importance and prevalence of gastritis, the relationship between intensities of gastritis and bacterial colonization is investigated; and the necessity of specific staining of Helicobacter pylori is determined according to the results. On the other hand, identifying the relationship between intensities of colonization and gastritis helps the clinician in more effective treatment and posttreatment follow-ups (response rate to antibiotic therapy).

\section{Methodology}

In this cross-sectional study, endoscopic gastric biopsy samples of 544 patients referring to the Razi laboratory of Hamadam city in Iran were studied between 2014-2015 regardless of their occupation, education, economic and social status, and food habits. The mentioned patients complained symptoms of dyspepsia for more than three months and had not used antibiotics during the past month; they had no history of surgery on the gastrointestinal tract and did not have any underlying disease other than dyspepsia. To determine the colonization rate of $H$. pylori and other pathological findings, Giemsa and $\mathrm{H} \& \mathrm{E}$ stains were, respectively, used. In this study, we used the Sydney system grading of chronic gastritis for grading of chronicity, activity, and $H$. pylori density [6]. Scattered organisms covering less than one third of the surface are regarded as mild colonization; large clusters or a continuous layer over two thirds of the surface is graded as severe; intermediate numbers are mentioned as moderate colonization. The normal number of gastric mucosal mononuclear cells in the lamina propria is defined as a maximum of 2 to 5 lymphocytes, plasma cells, and macrophages per high-power field ( $\times 40$ objective). Mild chronic inflammation is defined as a mild increase of inflammatory infiltration, predominantly plasma cells, within the lamina propria in a patchy, loose distribution without destruction or involvement of epithelium using $\times 10$ objective lens to identify mononuclear clusters. Dense lymphoplasma cell infiltration of the lamina propria with or without lymphoid follicles, easily identifiable on $\times 4$ objective lens, with infiltration and destruction of epithelium is regarded as severe chronic inflammation. Intermediate status is mentioned as moderate degree. Activity of gastritis is defined as neutrophilic infiltration of the lamina propria, pits, or surface epithelium. Less than one third of pits and surface infiltration are regarded as mild; one third to two thirds are graded as moderate; more than two thirds are regarded as severe.
TABLE 1: Frequency distribution of the activity level of inflammation among the subjects participating in the study.

\begin{tabular}{lcc}
\hline Activity level of inflammation & Frequency & Percentage \\
\hline Without activity & 166 & 30.51 \\
Mild & 201 & 36.95 \\
Moderate & 66 & 12.13 \\
Severe & 111 & 20.42 \\
Total & 544 & 100 \\
\hline
\end{tabular}

Atrophic changes are defined as loss of specialized glands from either the antrum or corpus. Metaplastic epithelium is recognized morphologically by the presence of goblet cells, absorptive cells, and cells resembling colonocytes.

Patient information was entered into the checklist and analyzed with SPSS16. The significance level was determined as 0.05 ; amounts less than five hundredths were considered statistically significant. The statistical test that is used was chi-square.

\section{Findings}

The average age of the participants in the study was 43.62 years with a standard deviation of 16.74 with a minimum and maximum age of 1 and 87 years (with an age range of 86 years), respectively. The average age for men and women was 45.87 and 41.50 years, respectively.

Of the 544 patients with $H$. pylori infection, $51.84 \%$ were female and there was no statistically significant relationship between gender and $H$. pylori infection $(P$ value $=0.67)$. Individuals with mild, moderate, and severe $H$. pylori infection were divided by gender in which $52 \%, 54 \%$, and $43 \%$ of them are female, respectively. Despite the higher percentage of women in the mild and moderate infected groups, there was no statistically significant relationship between gender and intensity of $H$. pylori infection $(P$ value $=0.56)$.

Among 544 subjects, 47 (8.64\%) patients had no gastritis, 203 (37.32\%) had mild gastritis, 278 (51.10\%) suffered from moderate gastritis, and 16 (2.94\%) had severe gastritis.

Most of the participants were in the mild activity group and the moderate activity group included the least number of patients (Table 1).

$15.07 \%$ the participants had superficial erosion. $4.23 \%$ had ulcer, $6.07 \%$ had atrophy, and $12.50 \%$ showed metaplasia. Also, $46.11 \%$ of the biopsies revealed lymphoid follicles.

$64.15 \%$ of the participants suffered from mild $H$. pylori infection, while $29.96 \%$ and $5.88 \%$ of the patients had moderate and severe $H$. pylori colonization, respectively.

In this study, patients with mild $H$. pylori colonization rates had the highest level of mild activity (33.52\%); in contrast, those with severe $H$. pylori colonization had the highest level of severe activity (43.75\%). There was a statistically significant relationship between the severity of $H$. pylori infection and activity $(P$ value $<0.001)$. Hence, the level of activity increases by increased intensity of colonization (Table 2).

In this study, the severity of chronic gastritis was mostly reported as mild (51.58\%) in people with mild $H$. pylori 
TABLE 2: Relationship between the level of activity and severity of $H$. pylori infection in patients with chronic gastritis.

\begin{tabular}{lcccccc}
\hline & & & & Activity intensity & \\
& & Lack of activity & Mild & Moderate & Severe & Total \\
\hline & Mild & $142(40.69 \%)$ & $117(33.52 \%)$ & $18(5.16 \%)$ & $72(20.63 \%)$ & $349(100 \%)$ \\
The severity of Helicobacter pylori colonization & Moderate & $24(14.72 \%)$ & $73(44.79 \%)$ & $41(25.15 \%)$ & $25(15.34 \%)$ & $163(100 \%)$ \\
& Severe & $0(0.00 \%)$ & $11(34.38 \%)$ & $7(21.88 \%)$ & $14(43.75 \%)$ & $32(100 \%)$ \\
& Total & $166(30.51 \%)$ & $201(36.95 \%)$ & $66(12.13 \%)$ & $111(20.40 \%)$ & $544(100 \%)$ \\
\hline
\end{tabular}

$P$ value (chi-square) $<0.001$.

TABLE 3: Relationship between severity of chronic gastritis and intensity of $H$. pylori infection.

\begin{tabular}{lcccccc}
\hline & & & \multicolumn{4}{c}{ Chronic gastritis severity } \\
& & Without gastritis & Mild & Moderate & Severe & Total \\
\hline \multirow{2}{*}{ The severity of Helicobacter pylori colonization } & Mild & $46(13.18 \%)$ & $180(51.58 \%)$ & $121(34.67 \%)$ & $2(0.57 \%)$ & $349(100 \%)$ \\
& Moderate & $1(0.61 \%)$ & $21(12.88 \%)$ & $136(83.44 \%)$ & $5(3.07 \%)$ & $163(100 \%)$ \\
& Severe & $0(0.00 \%)$ & $2(6.25 \%)$ & $21(65.63 \%)$ & $9(28.13 \%)$ & $32(100 \%)$ \\
& Total & $47(8.64 \%)$ & $203(37.32 \%)$ & $278(51.10 \%)$ & $16(2.94 \%)$ & $544(100 \%)$ \\
\hline
\end{tabular}

$P$ value (chi-square) $<0.001$.

colonization. In contrast, $93.76 \%$ of people with severe $H$. pylori colonization suffered from moderate and severe chronic gastritis. There was a significant statistical relationship between the incidence of $H$. pylori infection and the severity of chronic gastritis. As shown in Table 3, the severity of chronic gastritis increases with the increasing intensity of $H$. pylori colonization.

As Table 4 shows, there is a significant statistical relationship between the intensity of $H$. pylori colonization and histopathological findings including intestinal metaplasia, atrophy, and lymphoid follicle formation. However, no significant relationship was found between the intensity of colonization and ulcer formation. That is, by increasing the degree of colonization, likelihood of the formation of metaplasia, atrophy, and lymphoid follicle formation is increased.

\section{Discussion}

Regarding its numerous complications affecting the upper gastrointestinal tract and its high prevalence in developing countries including our country, correct diagnosis and proper treatment of $H$. pylori infection are a necessity. The aim of this study was to investigate the relationship between the intensity of $H$. pylori colonization in gastric mucosa and the severity of gastric mucosal pathological findings in biopsy specimens.

In this study, more than $90 \%$ of people infected with $H$. pylori experienced varying degrees of gastritis, with moderate gastritis (51.10\%) having the highest incidence. In the study conducted by Hashemi et al. in southern Iran (2006), 70\% of people infected with $H$. pylori suffered from chronic gastritis and in another study in Saudi Arabia, this rate was 60 percent $[7,8]$. Compared to these studies, the incidence of chronic gastritis secondary to $H$. pylori infection is higher in our study due to the evaluation of symptomatic patients.
The findings of this study showed that there is a dose response relationship between $H$. pylori colonization and activity such that in mild colonization, only $20.63 \%$ of the samples had severe activity while in intense colonization of $H$. pylori, $43.75 \%$ of the samples had severe activity. In a study by Alagoz et al. in Turkey (2002), it was shown that there is a positive correlation between the level of colonization and activity (Pearson correlation coefficient $=0.72$ ) which was confirmed by the present study as well [9].

Our study also showed that there is a direct relationship between the severity of $H$. pylori infection and the degree of chronic gastritis. Hence, if the level of colonization is mild, the chance of severe chronic gastritis among the subjects was only $0.57 \%$ while this chance was about 28 percent among those with severe colonization.

In a study conducted by Ardakani and Mohammadizadeh in Isfahan (2006) on 272 samples of gastric biopsy, there was no significant relationship between the density and volume of H. pylori and the severity of chronic gastritis activity [3].

In a study by Yakoob and Hussainy in Pakistan (2010), there was a statistically significant correlation between the intensity of $H$. pylori colonization and chronic gastritis activity [10].

This relationship was not found in the study conducted by Park et al. in Korea, which could be due to the genetic differences, nutritional habits, and environmental factors between the two study populations [11].

The study of Choudhary et al. in Nepal (2001) entitled "Correlation of H. Pylori density with grading of chronic gastritis" conducted on 251 patients showed a lack of correlation [12]. However, as already mentioned, a coherent correlation was observed in our study.

In this study, there was no statistically significant difference between the levels of $H$. pylori infection by gender. Albeit, the prevalence of different degrees of $H$. pylori 
TABLE 4: Relationship between histopathological findings and severity of $H$. pylori infection in patients with chronic gastritis.

\begin{tabular}{|c|c|c|c|c|c|c|}
\hline \multicolumn{3}{|c|}{ The severity of Helicobacter pylori colonization } & \multirow{2}{*}{$\frac{\text { Mild }}{30(8.60 \%)}$} & \multirow{2}{*}{$\begin{array}{c}\text { Moderate } \\
32(19.63 \%)\end{array}$} & \multirow{2}{*}{$\begin{array}{c}\text { Severe } \\
6(18.75 \%)\end{array}$} & \multirow{2}{*}{$\frac{P \text { (chi-square) }}{0.001}$} \\
\hline \multirow{8}{*}{ Histopathological findings } & \multirow{2}{*}{ Metaplasia } & Yes & & & & \\
\hline & & No & $319(91.40 \%)$ & $131(80.37 \%)$ & $26(81.25 \%)$ & 0.001 \\
\hline & \multirow{2}{*}{ Atrophy } & Yes & $13(3.72 \%)$ & $15(9.20 \%)$ & $5(15.63 \%)$ & \multirow{2}{*}{0.004} \\
\hline & & No & $336(96.28 \%)$ & $148(90.80 \%)$ & $27(84.38 \%)$ & \\
\hline & \multirow{2}{*}{ Ulcer } & Yes & $10(2.87 \%)$ & $10(6.13 \%)$ & $3(9.38 \%)$ & \multirow{2}{*}{$0.07^{*}$} \\
\hline & & No & $339(97.13 \%)$ & $153(93.87 \%)$ & $29(90.63 \%)$ & \\
\hline & \multirow{2}{*}{ Lymphoid follicle } & Yes & $106(34.53 \%)$ & $100(61.73 \%)$ & $25(78.13 \%)$ & \multirow{2}{*}{0.001} \\
\hline & & No & $201(65.47 \%)$ & $62(38.27 \%)$ & $7(21.88 \%)$ & \\
\hline
\end{tabular}

${ }^{*}$ Not significant.

infection was higher in women except for severe infection which may be due to frequent visits by women to the clinic for treatment. In this study, $48.16 \%$ of the subjects were male, while the female population was $51.84 \%$. In most previous studies, there was no significant relationship between $H$. pylori infection and gender. For example, the results of a study conducted by Everhart et al. in 2000 in the United States showed that there is no statistically significant difference between two genders [13]. Findings of our study were consistent with the study by Klein et al. (1994) regarding gender differences [14].

In this study, the percentage of intestinal metaplasia observed in 68 subjects was $12.5 \%$, which is higher than the percentage reported by Fakher.Yasseri H. (11.3\%) [15]. In the study of Zhang et al. in Japan (2005), 37\% of the subjects with chronic gastritis had metaplasia [16].

The results of a study conducted in Iraq in 2011 indicated a higher prevalence of intestinal metaplasia compared to our study. In this study, the prevalence of metaplasia was $15 \%$, while this rate was $12.5 \%$ in our study [17]. Perhaps the high percentage of metaplasia in other studies is due to genetic differences, nutritional habits, and differences in the duration of gastritis.

In this study, there was no significant relationship between $H$. pylori colonization and ulcer development.

In our study, only 23 cases (23.24\%) suffered from ulcer among pathology specimens. There was no statistically significant relationship between ulcer and $H$. pylori colonization severity. In mild, moderate, and severe colonization, $2.87 \%, 6.13 \%$, and $9.38 \%$, respectively, had ulcer.

In a study by Zhang et al. in Japan in 2005, it was concluded that there is a strong correlation between the amount of $H$. pylori colonization and ulcer [16]. This difference might be due to the small number of people with ulcer in our study.

The results of our study showed that 33 patients $(6.07 \%)$ had atrophic disease. Among pathologic specimens, 231 cases $(46.11 \%)$ of lymphoid follicles were observed. In both conditions, there was a statistically significant difference between the intensity of colonization in those with and without such complications. The study results of Kuipers et al. (1995) in the Netherlands confirmed the findings of our study, suggesting that $H$. pylori infection is an important risk factor for atrophy and the formation of lymphoid follicles [18].
One of the strengths of our study is its large sample volume compared to other studies. If this study was simultaneously conducted at multicenters, it could have a higher ability to conclude on hypotheses.

\section{Conclusion}

According to the present study, with increasing intensity of H. pylori colonization, chronicity and activity of gastritis and its complications and the consequent financial burdens imposed on the health system may increase. Correct treatment of $H$. pylori infection can be useful in preventing chronic gastritis and its complications. Therefore, it seems that reduction of $H$. pylori colonization should be considered as a health goal.

\section{Disclosure}

This paper was extracted from a $\mathrm{PhD}$ thesis by Parvin Akbari [19].

\section{Conflicts of Interest}

The authors declare that they have no conflict of interest.

\section{Acknowledgments}

The authors would like to thank the personnel of the Razi Pathobiology Laboratory in Hamadan, Iran, for their contributions in this study and for providing regular filing in their pathology archive. This project was funded with support from the Hamadan University of Medical Sciences, Hamadan, Iran.

\section{References}

[1] S. Amouian, N. TayebiMeybodi, and F. Farhadi, "Assessment of efficiency and reproducibility of Sydney system in chronic gastritis grading," Journal of Birjand University of Medical Sciences, vol. 15, no. 1, pp. 52-58, 2008.

[2] D. Longo, L. Jemson, and A. Favesi, Harrison's Principle of Internal Medicine, McGraw-Hill, New York, 18th edition, 2012.

[3] A. Ardakani and F. Mohammadizadeh, "The study of relationship between Helicobacter pylori density in gastric mucosa 
and the severity and activity of chronic gastritis," Journal of Research in Medical Sciences, vol. 11, no. 4, p. 282, 2006.

[4] M. F. Dixon, R. M. Genta, J. H. Yardley, and P. Correa, "Classification and grading of gastritis. The updated Sydney system. International workshop on the histopathology of gastritis, Houston 1994," The American Journal of Surgical Pathology, vol. 20, no. 10, pp. 1161-1181, 1996.

[5] M. Ashton-Key, T. C. Diss, and P. G. Isaacson, "Detection of Helicobacter pylori in gastric biopsy and resection specimens," Journal of Clinical Pathology, vol. 49, no. 2, pp. 107111, 1996.

[6] M. Stolte and A. Meining, "The updated Sydney system: classification and grading of gastritis as the basis of diagnosis and treatment," Canadian Journal of Gastroenterology, vol. 15, no. 9, pp. 591-598, 2001.

[7] M. Hashemi, M. Rahnavardi, B. Bikdeli, and M. D. Zahedani, "H pylori infection among 1000 southern Iranian dyspeptic patients," World Journal of Gastroenterology, vol. 12, no. 34, pp. 5479-5482, 2006.

[8] A. Ayoola, H. Ageely, M. Gadour, and V. Pathak, "Prevalence of Helicobacter pylori infection among patients with dyspepsia in south-western Saudi Arabia," Saudi Medical Journal, vol. 25, no. 10, pp. 1433-1438, 2004.

[9] S. Alagoz, C. Turkay, and O. Yonem, "The relationship between Helicobacter pylori intensity and histopathological findings in cases with chronic gastritis and duodenal ulcer," The Turkish Journal of Gastroenterology, vol. 13, no. 2, pp. 98-102, 2002.

[10] M. Y. Yakoob and A. S. Hussainy, "Chronic gastritis and Helicobacter pylori: a histopathological study of gastric mucosal biopsies," Journal of the College of Physicians and Surgeons, vol. 20, no. 11, pp. 773-775, 2010.

[11] J. Park, M. K. Kim, and S. M. Park, "Influence of Helicobacter pylori colonization on histological grading of chronic gastritis in Korean patients with peptic ulcer," The Korean Journal of Internal Medicine, vol. 10, no. 2, pp. 125-129, 1995.

[12] C. Choudhary, U. Bhanot, A. Agarwal, and R. Garbyal, "Correlation of H. pylori density with grading of chronic gastritis," Indian Journal of Pathology \& Microbiology, vol. 44, no. 3, pp. 325-328, 2001.

[13] J. E. Everhart, D. Kruszon-Moran, G. I. Perez-Perez, T. S. Tralka, and G. McQuillan, "Seroprevalence and ethnic differences in Helicobacter pylori infection among adults in the United States," The Journal of Infectious Diseases, vol. 181, no. 4, pp. 1359-1363, 2000.

[14] P. D. Klein, R. H. Gilman, R. Leon-Barua, F. Diaz, E. O. Smith, and D. Y. Graham, "The epidemiology of Helicobacter pylori in Peruvian children between 6 and 30 months of age," American Journal of Gastroenterology, vol. 89, no. 12, pp. 2196-2200, 1994.

[15] Fakher.Yasseri H, "Determination of Helicobacter-pylori prevalence in histologic gastritis and intestinal metaplasia and related to age and sex study on 576 patients with nonulcer dyspepsia at endascopy department of Firozgar Hospital," Razi Journal of Medical Sciences, vol. 9, no. 30, pp. 379-387, 2002.

[16] C. Zhang, N. Yamada, W. YL, M. Wen, T. Matsuhisa, and N. Matsukura, "Comparison of Helicobacter pylori infection and gastric mucosal histological features of gastric ulcer patients with chronic gastritis patients," World Journal of Gastroenterology, vol. 11, no. 7, pp. 976-981, 2005.
[17] A. H. Bashar, H. Khesar, and A. Zobayda, "Prevalence of intestinal metaplasia in infectious and non infectious chronic gastritis," Scholars Journal of Applied Medical Sciences, vol. 2, no. 6C, pp. 3042-3045, 2014.

[18] E. J. Kuipers, A. M. Uyterlinde, A. S. Pena et al., "Long-term sequelae of Helicobacter pylori gastritis," The Lancet, vol. 345, no. 8964, pp. 1525-1528, 1995.

[19] P. Akbari, Determination of Relationship Between H. pylori Colonization Intensity and Gastritis Grading in Pathologic Reports in Hamedan Razi Labratory [Thesis for the Degree of Doctorate in Medicine], Hamadan University of Medical Sciences, Hamadan, 2015. 


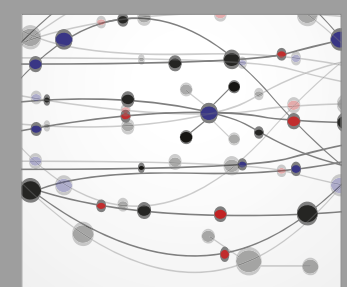

The Scientific World Journal
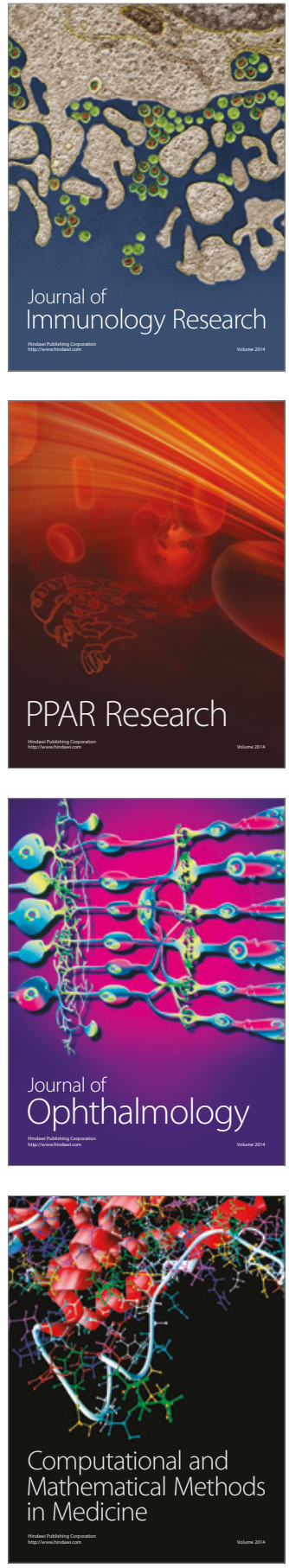

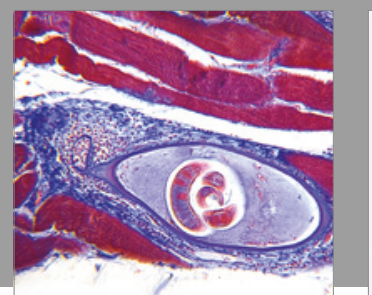

Gastroenterology Research and Practice
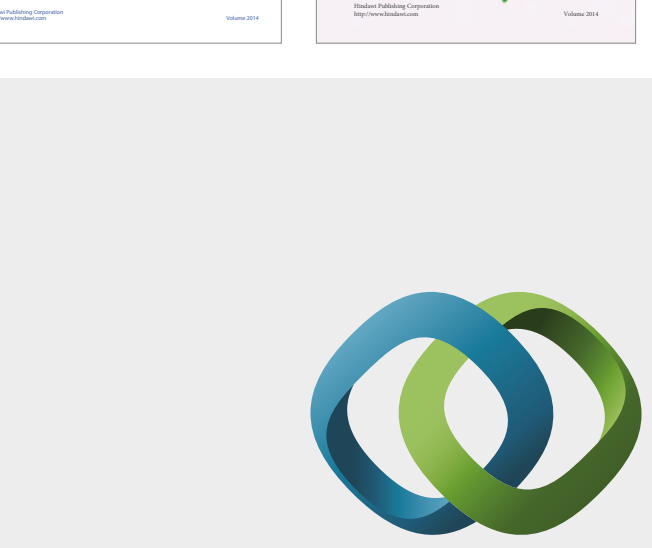

\section{Hindawi}

Submit your manuscripts at

https://www.hindawi.com
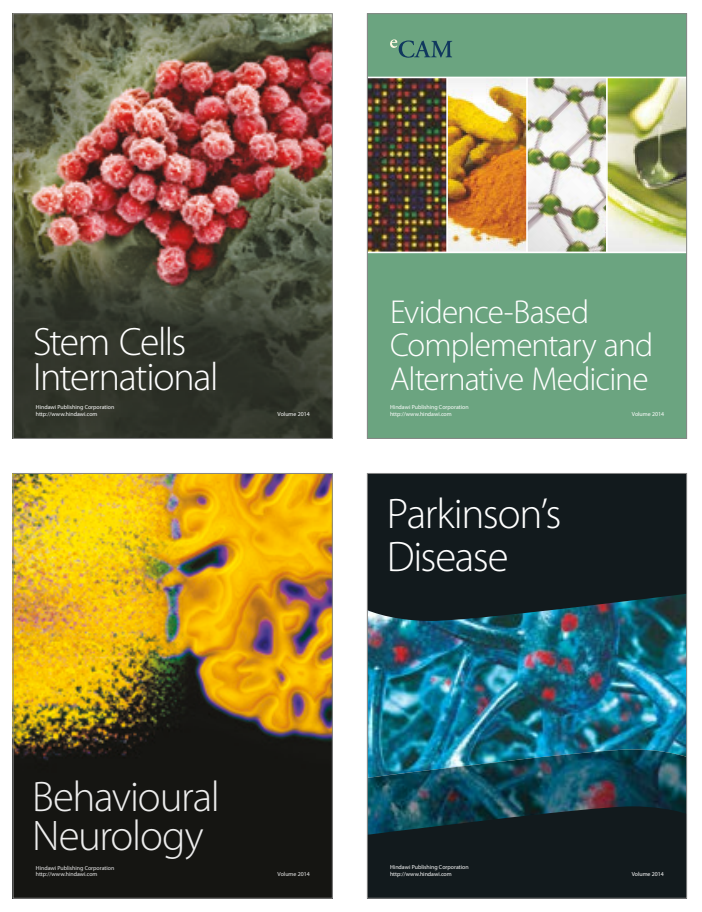
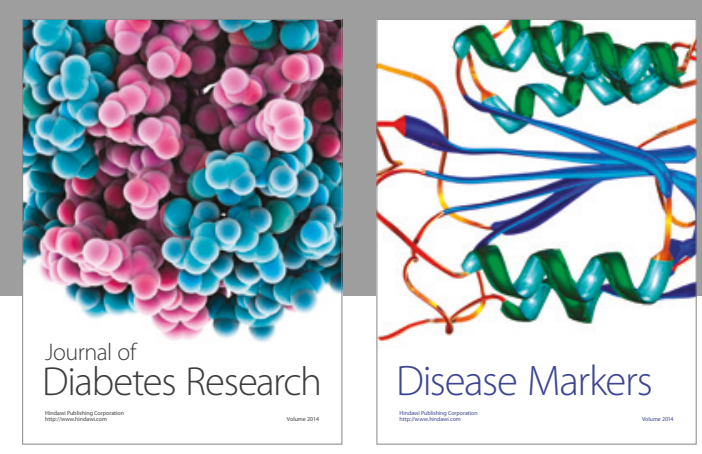

Disease Markers
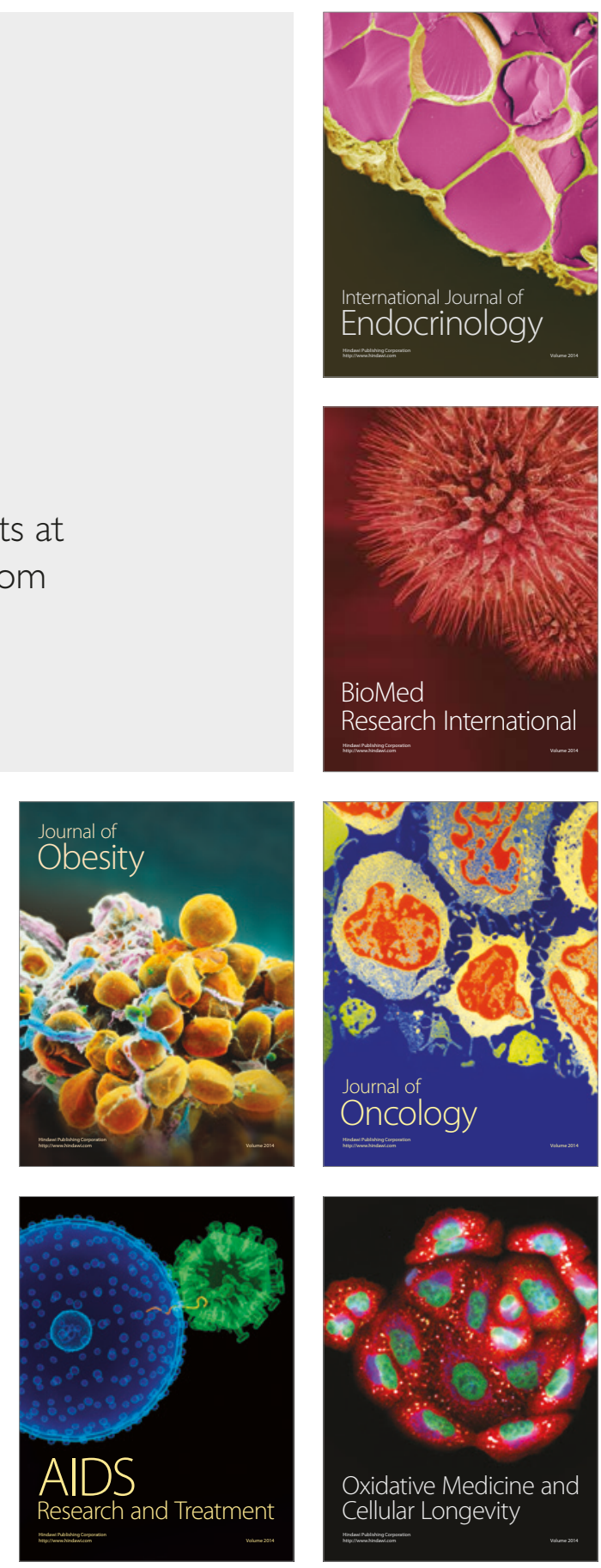